\title{
Brucellosis in Mirpur, Azad Kashmir Pakistan: a livestock threat for neighboring zones')
}

\author{
HADIA MUBEEN, IAHTASHAM KHAN*, MUHAMMAD HASSAN SALEEM, \\ RAHEELA AKHTAR ${ }^{* *}$, SHAHZAD ALI***, MUHAMMAD SAQIB ${ }^{* * * *}$, \\ AMAN ULLAH KHAN ${ }^{* * * *}$, SAJID UMAR******
}

\begin{abstract}
Department of Clinical Medicine and Surgery, **Department of Pathology, ${ }^{* *}$ Department of Wildlife and Ecosystem, Unive University of Veterinary and Animal Sciences, Lahore, Pakistan-54000 *Section of Epidemiology and Public Health, College of Veterinary and Animal Sciences, Jhang, Pakistan ${ }^{* * * *}$ Department of Clinical Medicine and Surgery, University of Agriculture, Faisalabad, Pakistan *****Friedrich Loeffler Institute, Jena, Germany ******Department of Pathobiology, Pir Mehr Ali Shah Arid Agriculture University Rawalpind, Pakistan
\end{abstract}

Mubeen H., Khan I., Saleem M. H., Akhtar R., Ali S., Saqib M., Khan A. U., Umar S. Brucellosis in Mirpur, Azad Kashmir Pakistan: a livestock threat for neighboring zones

Summary

The objective of the present study was to determine the prevalence of brucellosis in household animals of Mirpur, Azad Kashmir due to its geographic importance. A total of 360 blood samples of cattle, buffaloes, sheep and goats were initially screened through Rose Bengal Plate test (RBPT) and then positive samples were subjected to Enzyme Linked Immunosorbent Assay (ELISA) for confirmation and quantification of antibody titers. Molecular confirmation of serologically positive samples was performed by real time polymerase chain reaction (PCR). RBPT and ELISA showed a total of $8.6 \%$ and $6.87 \%$ positive samples respectively. The species wise seropositivity by RBPT was greater in cattle followed by buffaloes, goats and sheep. Similarly ELISA showed more seropositivity in cattle than buffaloes, while sheep and goats were negative for brucellosis by ELISA. RT-PCR revealed $100 \%$ samples positive for Brucella abortus by species specific PCR. This study revealed the presence of Brucella abortus in Mirpur for the first time. Since brucellosis is listed in transboundary diseases, its presence in this geographically important region could be a potential threat for neighboring countries.

Keywords: Brucella abortus, ELISA, PCR, RBPT, Serum

Brucellosis is a most common livestock and public health issue in terms of abortions, repeat breeding, reduced milk production, birth of weak calves and treatment cost. It is widespread in 86 countries of the world, particularly in developing countries, and imparts a wide range of socio-economic impacts especially where dairy products and livestock breeding are major sources of income $(3,4)$.

In addition, the significance of this disease cannot be neglected as an occupational risk for farmers, shepherds, butchers, laboratory workers and veterinarians. As brucellosis causes dangerous consequences, like the prevention of trading animals and animal products, and also inhibits public and economic development of breeders in pastoral regions. Therefore the prevalence of this disease should be known for proper control

The authors are very grateful to the Federal German Foreign Office for financial research support through a research project "Brucellosis in Pakistan" project no Je-0113. and eradication programs, especially in border areas that can infect other countries by accidental or illegal movement of animals. In addition, purchasing diseased animals from an affected area may be risky. Keeping in view the above mentioned context the present study was designed to determine the presence of brucellosis in the animals (small and large ruminants) and humans of Mirpur, Pakistan. As Mirpur shares its borders with India the information obtained from this study could be beneficial for both countries. Therefore the main objective of this study was to determine the prevalence of brucellosis in household animals of Mirpur, Azad Kashmir, due to its geographic importance.

\section{Material and methods}

The present study was conducted in the three tehsils of district Mirpur, Azad Kashmir: i.e. Chakswari, Dodyal and Mirpur. Animals with a history of one or two abortions were included in the study. A total of 360 samples were collected 
from small holdings and commercial farms, which means a sum of 120 blood samples were collected from each subdivision of district Mirpur. These 120 blood samples were divided into four groups: i.e. Group $\mathrm{A}=$ cattle $(\mathrm{n}=30)$ Group $B=$ buffaloes $(n=30)$, Group $C=$ sheep $(n=30)$, Group $D=$ goats $(n=30)$. These samples were processed for serum collection at the Quality Operational Laboratory of the University of Veterinary \& Animal Sciences, Lahore. This study was approved by the ethical committee of the University of Veterinary \& Animal Sciences, Lahore Pakistan. Oral and written consent was taken from each farmer prior to blood sampling.

All serum samples $(n=360)$ were subjected to Rose Bengal Plate Test (RBPT) for initial screening for anti Brucella antibodies as described before (OIE. 2009). Results were compared with positive and negative control. Samples showing agglutination were declared as positive and the rest were negative. The serum samples that were positive with RBPT were further analyzed by indirect ELISA using the Brucella abortus antibody test kit (IDEXX Brucellose Serum X2 Ab Test, IDEXX Laboratories, Switzerland). Results were calculated using the ELISA reader at $450 \mathrm{~nm}$ wavelength. By the use of optical density (OD) value SP ratio was calculated. Samples having an SP ratio $80 \%$ or more than $80 \%$ were considered as positive. The colored reactions of enzymes linked with anti-antibody and substrate were measured by taking optical density at $450 \mathrm{~nm}$. Samples of OD value and OD value of positive and negative control were used for calculating the value of individual samples. Samples with equal or more than $80 \%$ value were considered as Brucella positive.

Confirmation of Brucella antigen in serum samples was performed by real time Polymerase chain reaction (PCR). Genomic DNA was extracted from serum samples by using "FavorPrep" $\mathrm{TM}$ Blood/ Cultured cells Genomic DNA extraction Mini kit" and protocol was followed as per manufacturer's instructions. All ELISA positive samples were subjected to real time PCR. First, Brucella genus identification was done by using Brucella genus/ universal primers and its respective probe. Those samples which were genus positive in $1^{\text {st }}$ PCR were subjected to a $2^{\text {nd }}$ PCR using Brucella species specific primers of Brucella abortus. All protocols and conditions were followed as per manufacturer's instruction by Thermo Fisher Scientific. For Brucella species identification, the primers and probe targeting bcsp31 gene
Tab. 2. Seroprevalence by RBPT and ELISA

\begin{tabular}{|l|c|c|c|c|c|}
\hline & Cattle & Buffaloes & Goats & Sheep & Total \\
\hline No & 90 & 90 & 90 & 90 & 90 \\
\hline RBPT + ve & 16 & 08 & 02 & 05 & 31 \\
\hline RBPT\% & $17.8 \%$ & $8.9 \%$ & $2.2 \%$ & $5.6 \%$ & $8.6 \%$ \\
No. of samples taken randomly & 24 & 32 & 38 & 35 & 129 \\
RBPT + ve samples & 16 & 8 & 2 & 5 & 31 \\
Samples subjected to i-ELISA & 40 & 40 & 40 & 40 & 160 \\
ELISA + ve & 7 & 4 & 0 & 0 & 11 \\
ELISA + ve $\%$ & $17.5 \%$ & $10 \%$ & $0 \%$ & $0 \%$ & $6.87 \%$ \\
\hline
\end{tabular}

Tab. 3. Real time PCR results ct value for Brucella genus and Brucella abortus

\begin{tabular}{|c|c|c|c|c|c|}
\hline $\begin{array}{c}\text { Sample } \\
\text { No }\end{array}$ & $\begin{array}{c}\text { Brucella genus } \\
\text { RT-PCR ct value }\end{array}$ & $\begin{array}{c}\text { Brucella abortus } \\
\text { RT-PCR ct value }\end{array}$ & $\begin{array}{c}\text { Sample } \\
\text { No }\end{array}$ & $\begin{array}{c}\text { Brucella genus } \\
\text { RT-PCR ct value }\end{array}$ & $\begin{array}{c}\text { Brucella abortus } \\
\text { RT-PCR ct value }\end{array}$ \\
\hline 1 & 28.14 & 31.07 & 17 & N/A & \\
2 & N/A & & 18 & N/A & \\
\hline 3 & 34.48 & 37.45 & 19 & N/A & \\
4 & N/A & & 20 & 37.98 & 40.12 \\
\hline 5 & 37.43 & 29.00 & 21 & 40.29 & 37.87 \\
6 & N/A & & 22 & N/A & \\
\hline 7 & 39.27 & 32.76 & 23 & N/A & \\
\hline 8 & N/A & & 24 & 29.62 & 34.73 \\
\hline 9 & 37.08 & 32.34 & 25 & N/A & \\
10 & N/A & & 26 & 38.45 & 32.12 \\
\hline 11 & N/A & & 27 & N/A & \\
12 & N/A & & 28 & N/A & \\
\hline 13 & 37.22 & 32.12 & 29 & 30.50 & 31.62 \\
\hline 14 & N/A & & 30 & N/A & \\
\hline 15 & N/A & & 31 & N/A & \\
\hline 16 & N/A & & & &
\end{tabular}

(Gene Bank accession number M20404) were used. The nucleic acid targets for $B$. abortus identification are similar to those previously described (5). The B. abortus primers and probe set targets the specific insertion of an IS711 element downstream of the alkB gene (GenBank accession number AF148682).

$B$. abortus targets IS711 reverse primer, while the forward primers target alkB (B. abortus). The B. abortus TaqMan probes target the alkB gene. Sequences of above used primers and probes are given in Table 1.

\section{Results and discussion}

This study was undertaken to assess the presence of brucellosis in household ruminants (sheep, goat, cattle and buffalo) in the Mirpur district of Azad Kashmir. 
The selected areas showed the significant prevalence of brucellosis caused by Brucella abortus.

Out of the total of 360 serum samples only 31 samples were positive by Rose Bengal Plate Test (RBPT) and 11 were positive by iELISA (Tab. 2). The higher number of positive results by RBPT than ELISA could be due to more false positive results by RBPT as described before (1).

All RBPT and ELISA positive samples were further tested by real time PCR and 11 were positive for $B$. abortus. After each real time PCR few positive PCR products were run on agarose gel along with positive and negative controls for confirmation of PCR products and to find the amplicon size. Among 11 positive samples 7 samples have cycle thresh hold value from 30 to 39 , which indicate they were weak positive or have a too small amount of genomic DNA of Brucella. Two samples had ct values from 39 to 43, which indicate that those were very weak positive. Two samples had ct values from 25 to 30, showing that they are strong positive. For Brucella abortus one sample was strong positive, nine were weak positive, one was very weak positive (Tab. 3). The present study showed more positive cases of brucellosis in cattle than buffaloes. This is in agreement with previous studies (6) that also reported a greater prevalence of brucellosis in cattle than buffaloes in Quetta, Pakistan. This may be attributed to greater brucellosis resistance in buffaloes than cattle (2).
Interestingly, only Brucella abortus was found in all animals including sheep and goats. The unusual presence of this pathogen in small ruminants shows the cross species transmission of Brucella and cannot be ignored for brucellosis control programs. Another important piece of information provided by this study was the presence of more Brucella positive cases in sheep than goats. This could be due to the differential physiology of sheep and goats that make them resistant or susceptible to brucellosis.

\section{References}

1.Ahmed I. M., Khairani-Bejo S., Hassan L., Bahaman A. R., Omar A. R. Serological diagnostic potential of recombinant outer membrane proteins (rOMPs) from Brucella melitensis in mouse model using indirect enzymelinked immunosorbent assay. BMC Vet. Res. 2015, 11, 1-10.

2. Borriello G., Capparelli R., Bianco M., Fenizia D., Alfano F., Capuano F., Ercolini D., Parisi A., Roperto S., Iannelli D.: Genetic resistance to Brucella abortus in the water buffalo (Bubalus bubalis). Infect. Immun. 2006, 74, 2115 -2120 .

3. Maadi H., Moharamnejad M., Haghi M.: Prevalence of brucellosis in cattle in Urmia, Iran. Pak. Vet. J. 2013, 31, 81-82.

4. Mukhtar F.: Brucellosis in a high risk occupational group: seroprevalence and analysis of risk factors. JPMA 2010, 60, 1031-1040.

5. Redkar R., Rose S., Bricker B., DelVecchio V.: Real-time detection of Brucella abortus, Brucella melitensis and Brucella suis. Mol. Cell. Probes. 2001, 15, 43-52.

6. Shafee M., Rabbani M., Sheikh A. A., Razzaq A.: Prevalence of bovine brucellosis in organized dairy farms, using milk ELISA, in Quetta City, Balochistan, Pakistan. Vet. Med. Int. 2011, 2011, 1-3.

Corresponding author: Dr. Raheela Akhtar, Assistant Professor, PhD (UVAS, U.S.A), Department of Pathology, Faculty of Veterinary Sciences, University of Veterinary and Animal Sciences, Lahore, Pakistan. 54000; e-mail: raheela.akhtar@uvas.edu.pk 